\title{
Study of protein-protein interactions of human E3 ubiquitin ligase NEDD4-2 using fluorescence spectroscopy
}

\author{
Rohit Joshi ${ }^{1,2}$, Tomas Obsil ${ }^{1,2}$ and Veronika Obsilova ${ }^{1}$ \\ ${ }^{1}$ Department of Structural Biology of Signaling Proteins, Division BIOCEV, Institute of Physiology of the Czech Academy of Sciences, \\ 25250 Vestec, Czech Republic. \\ ${ }^{2}$ Department of Physical and Macromolecular Chemistry, Faculty of Science, Charles University, 12843 Prague, Czech Republic
}

\section{Rohit.Joshi@fgu.cas.cz}

Neural precursor cells expressed developmentally downregulated protein 4- 2 (Nedd4-2) plays a key role in the ubiquitination process, which leads to the endocytosis and degradation of its downstream target molecules such as membrane proteins. Nedd4-2 belongs to the HECT ubiquitin ligase family, which regulates signal transduction through interaction with other proteins including 143-3 proteins. 14-3-3s are evolutionarily conserved proteins, which negatively regulate Nedd4-2 in cAMP- dependent manner through phosphorylation by protein kinase A (PKA). This regulation is performed by providing scaffolding for Nedd4-2, thereby preventing the interaction with Nedd4-2 and other membrane proteins. Though this is known, the molecular mechanism of this regulation remains unknown and is under scientific scrutiny. We aim to understand the structural and functional basis of 14-3-3 mediated regulation of Nedd4-2 using combined structural biology and biophysical approaches such as fluorescence spectroscopy, protein crystallography and chemical crosslinking coupled with mass spectroscopy

Possible mechanism of the 14-3-3 mediated inhibition of pNedd4-2 includes stabilization of inactive conformation of Nedd4-2 in which, HECT and $\mathrm{C} 2$ domains are involved in the intramolecular interaction and steric masking of WW domains surfaces. To test this hypothesis, we performed the time resolved fluorescence spectroscopy measurements using phosphorylated Nedd4-2 variants labelled by extrinsic fluorophore and monitor their interaction with 14-3-3 protein. Fluorescence spectroscopy provided basic information on the dynamics of the interaction between Nedd4-2 ligase and 14-3-3 protein. Measuring of rotational correlation time and determination of the mean lifetime values of excited fluorophore in Nedd4-2 alone and in the complex with 14-3-3 protein (containing no Cys residues) allows us to trace the microenvironment of one particular cysteine amino acid, which is located at different positions within Nedd4-2 construct.

We also crystallized the complex of 14-3-3 $\gamma \Delta \mathrm{C}$ with the peptide containing phosphorylated $\mathrm{Ser}^{342}$, solved its structure using molecular replacement and refined it at $1.61 \AA$ resolution.

[1] J. A. Manning and S. Kumar, Trends Biochem. Sci. 43, (2018), 635-647.

[2] P. Goel, J. A. Manning, and S. Kumar, Gene, 557, (2015), 1-10.

[3] Nagaki K, Yamamura H, Shimada S, Saito T, Hisanaga S, Taoka M, Isobe T, Ichimura T, Biochemistry, 45, (2006), 6733-40.

[4] Ichimura T, Isobe T, J Biol Chem., 280, (2005), 13187-94.

This study was supported by the Czech Science Foundation (Projects 20-00058S), the Czech Academy of Sciences (Research Projects RVO: 67985823 of the Institute of Physiology) and by Grant Agency of Charles University (Project No. 348421).

Keywords: 14-3-3 protein:Nedd4-2; fluorescence Spectroscopy, protein- protein interactions, Protein crystallization 